\title{
MAQUINISTAS
}

\section{LA PARTICIPACIÓN INFANTIL A TRAVÉS DEL JUEGO}

\section{MACHINISTS}

CHILD PARTICIPATION THROUGH PLAY

Nicolás Knell | nicolasandresknell@gmail.com

Rodolfo Rodriguez | rre92rodolfo@gmial.com

Facultad de Bellas Artes. Universidad Nacional de La Plata. Argentina

Martina Iribarne | martinairibarne@yahoo.com.ar

Facultad de Trabajo Social. Universidad Nacional de La Plata. Argentina

Recibido: 13/2/2019| Aceptado: 6/5/2019

\section{RESUMEN}

Maquinistas es un juego de tablero que se propone favorecer la participación de los Niños, Niñas, Adolescentes y Jóvenes (NNAyj) en los procesos de salud-enfermedadatención-cuidado que los involucran, propiciando la toma de decisiones, el desarrollo de estrategias y el fortalecimiento de proyectos vitales. Para su planificación se tomó como población de trabajo a aquellos NNAyj que necesitan soportes nutricionales para vivir, en particular, quienes realizan Nutrición Parenteral Domiciliaria en el Servicio de Nutrición del Hospital Interzonal de Agudos Especializado en Pediatría (HIAEP) Sor María Ludovica.

\section{Palabras clave}

Juego; políticas públicas; diseño; salud; niñez

\begin{abstract}
Maquinistas [Machinists] is a board game that aims to promote the participation of Children, Girls, Adolescents and Youth (NNAyj) in the health-disease-care-care processes that involve them, encouraging decision-making, strategy development and the strengthening of vital projects. For its planning, those children and children who need nutritional support to live were taken as working population, in particular, those who perform Home Parenteral Nutrition in the Nutrition Service of the Interzonal Hospital of Acute Specialized in Pediatrics (HIAEP) Sr. María Ludovica.
\end{abstract}

\section{KEYWORDS}

Game; public politics; design; health; childhood 
Maquinistas es un proyecto de trabajo que nace de la inquietud colectiva e interdisciplinaria por repensar las formas de asistencia sanitaria y la participación de los Niños, Niñas, Adolescentes y Jóvenes (NNAyJ) que requieren de atención de alta complejidad y asistencia de tecnología para el desarrollo de la vida. La propuesta ha sido diseñada específicamente para aquellos NNAyj que realizan Nutrición Parenteral Domiciliaria en el Servicio de Nutrición del Hospital Interzonal de Agudos Especializado en Pediatría (HIAEP) Sor María Ludovica, aunque es posible utilizarla para la atención sanitaria de otros NNAyj con tratamientos complejos y prolongados.

De la evaluación diagnóstica que realizamos con el equipo de salud, los NNAyj asistidos y sus grupos de referencia, supimos que, si bien gran parte de la atención se dirigía al trabajo directo con NNAyj - y que los mismos recibían buen trato y tenían un vínculo respetuoso por parte del equipo-, en un gran número de ocasiones estos no comprendían la información que se les brindaba, por lo que las decisiones importontes eran tomadas por el equipo y/o sus familias. Observamos, además, una inquietud por parte de los actores involucrados en promover otras formas de participación infantil, aunque ello se limitaba a acciones e intervenciones puntuales.

Asimismo, analizamos los procesos de asistencia que desarrollaba el equipo de salud y las condiciones en las que realizaba su trabajo, ya que entendíamos que nuestra propuesta no solo debía poder ser utilizada por los NNAyj, sino también por quienes les brindaban asistencia, considerando que el dialogo y la toma de decisiones son actos relacionales.

De esta manera, comprendimos que era imprescindible analizar el modo en que los distintos actores interactúan en las estrategias de atención y cuidado asociadas con los procesos de salud-enfermedad, no solo en sus rasgos más formales, sino, fundamentalmente, en las trayectorias cotidianas de los NNAyj en este contexto sociohistórico. En este sentido, acordamos con Floreal Ferrara (1987) quien sostiene que la salud refiere a la capacidad singular y colectiva para luchar contra las condiciones que limitan la vida. Por consiguiente, no es posible comprender los procesos de enfermar y sanar como hechos aislados y univariables, independientes de los procesos sociales en los que se inscriben.

Así, la propuesta de construcción de un objeto lúdico que operara como soporte para la asistencia sanitaria estuvo directamente vinculada con la necesidad de construir un lenguaje común que tuviera en cuenta el contexto socio-histórico y las necesidades particulares de estos NNAyj, la diversidad de actores intervinientes, las trayectorias diferenciales y las experiencias previas, y que, al mismo tiempo, posibilitara generar diálogos no jerarquizados. Es decir, emergía como prioritaria la necesidad de establecer un intercambio comunicativo que propiciara la construcción colectiva de conocimiento, recuperando aspectos fundamentales como la toma de decisiones y el desarrollo de estrategias para el logro de objetivos.

Desde el punto de vista del diseño industrial, la inquietud respecto del modo en que ciertos objetos favorecen los procesos de asistencia sanitaria en tanto promueven la participación y la toma de decisiones $-\mathrm{y}$ propician alternativas terapéuticas 
respetuosas de los sujetos-, fue un aspecto transversal del proceso de trabajo que se describe.

\section{SOBRE LOS NNAYJ CON LOS QUE TRABAJAMOS}

Para el diseño y el desarrollo de esta propuesta trabajamos con NNAyj que requieren de soportes tecnológicos artificiales para el desarrollo de la vida. Nos centramos específicamente en quienes realizan Nutrición Parenteral Domiciliaria y se encuentran en seguimiento por la Unidad de Atención del Paciente en Domicilio (UAPD), perteneciente al Servicio de Nutrición y Dietoterapia del HIAEP Sor María Ludovica.

La mayoría de estos NNAyJ poseen insuficiencia intestinal, es decir, que su sistema digestivo no les permite digerir y absorber total o parcialmente los nutrientes necesarios para mantener la salud y el crecimiento. Como consecuencia de ello, requieren de la realización de un tratamiento complejo y prolongado que se denomina nutrición parenteral. Asimismo, la mayoría de estos NNAyj han sido diagnosticados luego del nacimiento, por lo que han crecido y se han socializado en directa vinculación con la enfermedad y con los distintos sistemas de asistencia.

El tratamiento que requieren no solo suele implementarse por un tiempo prolongado, sino que además implica que el NNAyj que lo realiza quede conectodo a él por aproximadamente doce horas diarias, procedimiento que debe repetirse durante varios días a la semana. Esta circunstancia, por un lado, limita la movilidad del NNAyj al radio establecido por su soporte, por lo que no puede realizar actividades que impliquen movimientos bruscos para el cuerpo mientras dura el proceso; por otro lado, lo condiciona a permanecer sentado o acostado más del tiempo habitual de sueño.

A pesar de que la UAPD diseña un plan de implementación singular para cada NNAyJ -que contempla su situación de salud, sus necesidades, requerimientos y posibilidades-, en todos los casos se requiere contar con: 1) un sistema de referencia y contrarreferencia sanitaria; 2) el acceso y la provisión del tratamiento; 3) las condiciones habitacionales adecuadas; 4) el entrenamiento y la organización de las tareas de cuidado; 5) el fortalecimiento de proyectos vitales. La forma en que se despliegan estrategias y se superan obstáculos en relación con la consecución de estos requerimientos y del egreso hospitalario son aspectos centrales para comprender el proceso de asistencia.

Los NNAyj que son asistidos provienen de distintas localidades de la provincia y/o del país, y han sido derivados de sus hospitales de referencia por no contar con los recursos edilicios, materiales y profesionales para la asistencia de alta complejidad asociada a esta problemática de salud. Muchas de sus familias ya poseen trayectorias previas de internación, aunque generalmente no han sido totalmente informadas respecto de la problemática de salud, del tipo de tratamiento requerido y de las condiciones necesarias para la implementación de soportes nutricionales domiciliarios 
(SND). Es decir, la mayoría de los NNAyj que son asistidos en el Servicio -y de sus familiares-, se interiorizan sobre cuestiones diagnósticas y de tratamiento mientras transitan por la internación. En este sentido, las trayectorias y los conocimientos compartidos son disimiles y están atravesados por múltiples determinantes.

Muchos de ellos han ocupado lugares pasivos no solo en las instituciones sanitarias, sino también en sus propios grupos familiares, por lo que han tenido poca autonomía respecto de su propio cuerpo y de las decisiones sobre la realización del tratamiento. Un número muy reducido de los NNAyj entrevistados habían podido transmitir a otros, aspectos de su situación de salud y/o del tipo de tratamiento que realizan, y preferían compartir sus inquietudes al respecto con otros pacientes y/o familiares.

\section{SOBRE EL JUEGO}

Maquinistas es un juego de estrategia en el que las y los participantes deben conseguir recursos para hacer crecer sus ciudades. Se juega sobre un territorio delimitado por vías ferroviarias y cada jugadora o jugador se moviliza a través de un tren a vapor que traslada los recursos desde las fábricas a las ciudades. A partir de elecciones tácticas y estratégicas las y los participantes deciden cómo establecer su ruta de viaje y administrar el combustible que poseen para conseguir los objetivos que les fueron asignados en las cartas.

El tablero, las piezas y el packoging están construidos íntegramente en madera de guatambú de seis y tres mm, ya que es un material de producción nacional, semirrígido y adecuado a las normas de bioseguridad hospitalaria, lo que posibilita su utilización sobre espacios irregulares - camas, mesas auxiliares, etcétera- mientras los NNAyj realizan el tratamiento. Se utilizó corte láser para reducir los costos de producción sin afectar la calidad. Asimismo, las cartas y el reglamento se realizaron en papel, y se utilizaron tinturas y barniz para el coloreado y acabado de las piezas. Cada juego posee: un tablero, ocho trenes, seis ciudades, doce bombas de agua, cinco fábricas, un contador general, un centro de reparaciones y un mazo de cartas.

Para el desarrollo de la dinámica del juego hicimos un trabajo de síntesis priorizando: 1) los aspectos centrales para la realización del SND; 2) las trayectorias singulares y colectivas de los NNAyj; 3) los elementos/objetos que utilizaban con mayor frecuencia; 4) los aspectos más significativos de su dinámica cotidiana incorporando categorías y analogías comunes-; 5) la ocurrencia de factores inesperados asociados a las complicaciones diagnosticas.

A lo largo de este proceso visualizamos que la cotidianeidad de estos NNAyJ estaba delimitada por ciclos - de conexión y desconexión-y por sistemas -articulación de condiciones para la implementación de Soportes Domiciliarios-. Asimismo, el ciclo témporo-espacial que une los periodos de conexión y desconexión al tratamiento debe ser respetado con rigurosidad, tiene una frecuencia cotidiana y opera como articulador del sistema de atención.

\section{TABLEROS \\ N. ${ }^{\circ} 10$ | octubre 2019 | ISSN 2525-1589}


A partir de estos criterios, consideramos que la construcción de un tablero en el que los jugadores se desplazaran por vías en trenes a vapor podía retomar la familiaridad de categorías y, al mismo tiempo, respetar un ciclo para la toma de decisiones - a través del sistema de carga de combustible-. Por otra parte, se recreó el armado del sistema de atención -según los criterios mencionados por la UAPD-, a partir de la inclusión de las fábricas de recursos y las cartas de objetivos.

En este sentido, el territorio de juego es un mapa a habitar, solo delineado por vías de tren que se despliegan en vinculación o no con elementos demográficos naturales como lagos, montañas, bosques, etcétera. Cada jugador puede iniciar su partida en el lugar del tablero donde desee, contemplando únicamente posicionarse al costado de una vía. Al inicio de la partida cada jugador elige una pequeña ciudad, que opera como punto de apoyo, y un tren de carga, que le permite movilidad dentro del juego. El objetivo central consiste en hacer crecer la ciudad a partir de conseguir recursos que se encuentran en distintos lugares del territorio.

Es importante considerar que, a excepción del contador general, todas las demás piezas son móviles y se organizan de acuerdo a las elecciones y decisiones de los distintos jugadores. De esta manera, por turnos, cada jugador coloca fábricas de recursos a una distancia máxima de quince casilleros de sí mismo y bombas de agua a ocho. Como los trenes funcionan a vapor, cada participante debe evaluar su recorrido no solo en relación con la distancia que exista entre los recursos, sino también con el combustible que posee.

Cada ciudad necesita recursos distintos para crecer y, por ello, a cada jugador se le entregan cinco cartas al azar que le indican aquello que debe conseguir. Cada vez que alguien gira la perinola consume un turno de agua, por lo que para poder completar su objetivo debe recargar combustible en las bombas ubicadas en el tablero de acuerdo con sus necesidades. Asimismo, se requiere seguir el curso de la vía, es decir, los trenes no giran sobre su propio eje. Cada vez que alguien se queda sin agua y no puede completar su recorrido, pierde un turno y debe ubicarse en el taller de reparaciones que se encuentra en el centro del tablero - debajo del contador-, desde el cual vuelve a partir.

Este juego fue pensado desde la participación-acción, en tanto el objetivo didáctico del mismo se cumple al jugar, no al ganar 0 al finalizar. En este caso, poder valorar las necesidades propias y desplegar estrategias para el logro de ese objetivo -teniendo en cuenta para ello la necesidad de respetar un ciclo y de reconocerse en ese proceso-, promueve la reflexividad y propicia la participación en la asistencia sanitaria para decidir con autonomía.

Uno de los elementos centrales que guió la construcción estuvo vinculado con el interés por mantener una armonía entre el sentido lúdico y el sentido explicativo. Es decir, nos propusimos construir un juego que pudiera desarrollarse sin ningún tipo de explicación, pero que, si en ocasión de la atención quisiera utilizarse para elegir, tomar decisiones o superar obstáculos, fuera representativo y tuviera analogías respecto del proceso de asistencia y de las necesidades específicas para la realización del tratamiento. 


\section{PROCESO de tRABAJO}

Al inicio del proceso de trabajo observábamos que, si bien el equipo de salud poseía un amplio conocimiento sobre la temática y tenía un vínculo cercano con la población asistida, los recursos y las herramientas con los que contaban eran insuficientes como para transformar sus inquietudes en una propuesta de trabajo. Al pensar en las formas de superación de estas limitantes consideramos que la incorporación de la mirada del diseño industrial, no solo contribuyó al debate respecto de las formas y de las posibilidades de la participación infantil, sino que, además, favoreció la desnaturalización de categorías y de estrategias de trabajo que actuaban como obstáculos y/o como facilitadores en esos procesos de atención.

En este sentido, acordamos con Susana Cazzaniga (2002) cuando sostiene que aquello que posibilita la construcción de la interdisciplina en el campo de lo social no está dada por la especificidad de ninguna disciplina en particular, sino, más bien, por la posibilidad que las mismas tengan para encontrar un objetivo común y aportar su visión singular:

Si bien se pueden reconocer desde las diferentes disciplinas aspectos de incumbencia específica, la comprensión de las situaciones que se abordan merecen un análisis complejo que ponga en juego las especificidades de los saberes profesionales en clave de integralidad, para luego identificar las intervenciones particulares desde una perspectiva de complementariedad. Desde esta perspectiva no hay una disciplina particular que interviene en lo social, en todo caso habría una «mirada disciplinar» que se hace cargo de ciertos aspectos de lo social (s. p.).

Trabajar sobre la dimensión social del diseño no solo supuso un desafío en relación con nuestra participación en procesos de atención que usualmente son habitados por otras disciplinas - como la medicina, la psicología o el trabajo social-, sino que, además, implicó el desarrollo de una propuesta que pudiera mediar en las relaciones equipo sanitario-pacientes desde una perspectiva de complejidad -superando la tradicional inclusión de objetos biomédicos y/o tecnológicos como único soporte de la atención-.

Para ello tomamos como disparadores aquellos interrogantes que motorizaban nuestro análisis, entre otros: ¿a qué juegan y con quiénes juegan estos NNAyJ?, ¿cómo incide su situación de salud en sus juegos?, ¿sobre qué cosas quisieran saber/ decidir en su cotidianeidad?, ¿qué cosas/objetos les son familiares y cuáles les son extraños?, ¿cómo perciben los lugares que ocupan en sus relaciones sociales y qué estrategias despliegan?

A lo largo del proceso de trabajo, un desafío importante estuvo dado por la necesidad de superar nuestra propia jerarquización y valoración respecto de aquello 
que es importonte para la realización del tratamiento o para la atención de salud. Por ello, decidimos configurar las piezas de forma abstracta, no lineal y no valorativa - es decir, todas las piezas valen lo mismo-, para procurar que a priori no existieran desigualdades evidentes. En consonancia con ello, optamos por no nominarlas -así, por ejemplo, todos los recursos se llaman de la misma forma-, para poder trabajar sobre la propia elección de los NNAyj y sobre su consideración acerca de qué elementos, en cada caso, habían sido más difíciles o más fáciles de conseguir.

Por su parte, requirió de una revisión constante poder lograr una armonía entre el sentido educativo y el lúdico, para que el primero no se interpusiera y primara por sobre el segundo. Jugar es un acto constitutivo de ejercicio de derechos y, por consiguiente, que fuera divertido era un objetivo tan importante como que fuera útil. En nuestro caso, el objetivo didáctico del juego se cumple en el desarrollo de la dinámica, independientemente de que exista un ganador y/o de que la partida se finalice.

\section{REFLEXIONES FINALES}

Los tránsitos de los NNAyJ por el sistema sanitario han de ser entendidos en su singularidad, no solo en lo que refiere a la ocurrencia de la enfermedad y a las características de la atención de salud para esta población, sino, además, en relación con las posibilidades de participación en los procesos que los involucran, la explicitación de sus opiniones respecto de la aplicación o no de ciertos tratamientos, el trabajo sobre sus inquietudes, entre otras cuestiones.

En este caso, poder valorar las necesidades propias y desplegar estrategias para el logro de ese objetivo, teniendo en cuenta para ello la necesidad de respetar un ciclo y de reconocerse en ese proceso, ha promovido la capacidad reflexiva y la habilitación para decidir con autonomía. Nominar con la propia voz las dificultades y los facilitadores que ha tenido para decidir, la forma en que su propio movimiento lo ha ayudado o lo ha limitado, y el modo en el que las particularidades de este tratamiento han tenido es su cotidianeidad, son algunos de los logros centrales de su aplicación.

Dos desafíos centrales que se nos presentan actualmente son los vinculados a la aplicación y a la replicabilidad. Respecto de esto último, entendemos que en todas aquellas situaciones en las que sea posible encontrar un ciclo de tiempo/espacio y un sistema de organización para el desarrollo de la cotidianeidad, este juego podría ser aplicable. Ello no solo abarca otros tratamientos de salud complejos, sino también otros hechos y procesos sociales en general.

Por su parte, entendemos que la aplicación del juego como una dimensión más de la atención se encuentra condicionada por las lógicas biomédicas imperantes y por las condiciones adultocéntricas de organización del sistema, que operan como resistencia. No obstante, como contracara, observamos que muchos equipos sanitarios poseen un interés por generar mayor participación de los NNAyJ, mejorar 
los estándares de adherencia a los tratamientos de alta complejidad y/o garantizar la permanencia de pacientes en los sistemas sanitarios.

Finalmente, consideramos que la promoción de la participación infantil y el desarrollo de la autonomía de los NNAyj durante los procesos de atención que los involucran, ha mejorado la implementación de Dispositivos de Atención Domiciliaria. La implementación de Soportes de este tipo, por su parte, no solo impacta en la calidad de vida de los pacientes y de su grupo de referencia - en tanto favorece la inclusión-, sino que, también, incide en la operatividad y efectividad del sistema sanitario -en tanto permite prevenir y evitar las complicaciones propias de la hospitalización prolongada y bajar los costos de la atención-.

\section{REFERENCIAS}

Cazzaniga, S. (2002). Trabajo Social e interdisciplina: la cuestión de los equipos de salud. Morgen, (27). Recuperado de http://www.fhycs.unam.edu.ar/carreras/wp-content/ uploads/2017/03/Trabajo-Social-e-interdisciplina-autor-SUSANA-CAZZANIGA.pdf

Ferrara, F. (1987). Teoría social y salud. Ciudad Autónoma de Buenos Aires, Argentina: Catálogos. 